\title{
Vitaminas D e C para poedeiras na fase inicial de produção de ovos
}

\section{Daniely Salvador ${ }^{1}$, Douglas Emygdio de Faria ${ }^{2}$, Monica Roberta Mazalli ${ }^{1}$, Diogo Tsuyoshi Ito $^{1}$, Daniel Emygdio de Faria Filho ${ }^{3}$, Lúcio Francelino Araújo ${ }^{2}$}

\author{
1 Zootecnista, Mestre em Zootecnia. \\ 2 Departamento de Zootecnia da FZEA/USP/Pirassununga - SP. \\ ${ }^{3}$ Departamento de Zootecnia do NCA/UFMG/Montes Claros - MG.
}

RESUMO - Um experimento foi conduzido com o objetivo de avaliar os efeitos de duas fontes de vitamina D e três níveis de vitamina C sobre as características de desempenho, a qualidade interna e externa dos ovos, os níveis de cálcio total e iônico séricos e a resistência óssea de poedeiras. Foram utilizadas 288 galinhas da linhagem ISA Babcock B300 ${ }^{\circledR}$ com 23 semanas de idade, durante um período experimental de 12 semanas. Utilizou-se o delineamento inteiramente ao acaso em arranjo fatorial $2 \times 3$, com os fatores: fontes de vitamina D (colecalciferol e 25-hidroxicolecalciferol - 25(OH) $\mathrm{D}_{3}$ ) e de vitamina C (0, 100 e $200 \mathrm{ppm})$, totalizando seis tratamentos com oito repetições de seis aves. O nível basal de colecalciferol foi de $2.756 \mathrm{UI} / \mathrm{kg}$, correspondendo a $5,51 \mathrm{~g}$ do produto comercial Hy. ${ }^{\circledR} / \mathrm{t}$ de ração, como fonte de $25(\mathrm{OH}) \mathrm{D}_{3}$. Os fatores estudados não influenciaram o consumo de ração, a produção, o peso e a massa de ovos. Observou-se efeito da interação de fontes de vitamina sobre a conversão alimentar, que foi melhor quando utilizado metabólito 25(OH) $\mathrm{D}_{3}$ na ausência de vitamina C. Interações foram observadas para porcentagem de albúmen e porcentagem de gema, que aumentaram na presença de 200 ppm de vitamina C. O peso específico dos ovos, as concentrações de cálcio sérico, cinzas ósseas e a resistência à quebra não foram influenciadas pelas fontes de vitamina D e C. Houve interação para porcentagem e espessura de casca, cujos maiores valores foram obtidos com a suplementação de vitamina $\mathrm{C}$ na presença de $25(\mathrm{OH}) \mathrm{D}_{3}$. Em poedeiras na fase inicial de produção, a conversão alimentar é melhor com a utilização do $25(\mathrm{OH}) \mathrm{D}_{3}$ e a espessura e porcentagem de casca também melhoram com a utilização de $25(\mathrm{OH}) \mathrm{D}_{3}$ e a suplementação de vitamina C nas dietas (100 ou 200 ppm, respectivamente).

Palavras-chave: ácido ascórbico, desempenho, qualidade de ovos, resistência óssea, vitamina D

\section{Vitamins $D$ and $C$ for laying hens at the initial phase of egg production}

\begin{abstract}
An experiment was conducted to evaluate the influence of two vitamin D sources and three vitamin C levels on performance, internal and external egg quality, and bone strength characteristics. In addition, the total and ionic blood calcium concentrations, bone ash and calcium were determined. Two hundred and eighty eight 23-week-old ISA Babcock B-300 ${ }^{\circledR}$ laying hens were used during the 12 -week study in a $2 \times 3$ factorial arrangement: vitamin D sources (cholecalciferol and 25-hydroxycholecalciferol $\left.-25(\mathrm{OH}) \mathrm{D}_{3}\right)$ and vitamin $\mathrm{C}$ levels $(0,100$ and $200 \mathrm{ppm})$ resulting in six treatments with eight replicates of six hens each. The basal cholecalciferol level was 2,756 IU/kg, corresponding to 5.51 g $\mathrm{Hy} . \mathrm{D}^{\circledR} / \mathrm{t}$, as source of $25(\mathrm{OH}) \mathrm{D}_{3}$. Feed intake, egg production, egg weight and egg mass were not influenced by the treatments. An interaction was observed for feed conversion, which was improved when $25(\mathrm{OH}) \mathrm{D}_{3}$ was added without vitamin $\mathrm{C}$. Haugh unit and yolk index were not influenced, however, interactions were observed for albumen percent and yolk percent, which were improved when 200 ppm of vitamin C was supplemented. Egg specific gravity, serum calcium, bone ash and bone strength resistance were not influenced by the treatments. There was an interaction for shell percent and shell thickness, which were improved when vitamin $\mathrm{C}$ was added in association with $25(\mathrm{OH}) \mathrm{D}_{3}$. It was concluded, for laying hens at initial phase of egg production, that feed conversion is improved when $25(\mathrm{OH}) \mathrm{D}_{3}$ was the vitamin $\mathrm{D}$ source, and that shell thickness and shell percent are improved when the vitamin $\mathrm{D}$ source was $25(\mathrm{OH}) \mathrm{D}_{3}$ with diets supplemented with vitamin C (100 or 200 ppm, respectively).
\end{abstract}

Key Words: ascorbic acid, bone strength, egg quality, performance, vitamin D

\section{Introdução}

A obtenção de bons índices zootécnicos de desempenho e qualidade externa dos ovos geralmente resulta em sucesso para o setor produtivo de ovos comerciais. Reconhece-se que a idade é determinante do desempenho e da qualidade interna e externa dos ovos (Faria et al., 1999a). Estima-se que a incidência de ovos quebrados 
seja de 6 a $8 \%$ do total de ovos produzidos, o que tem como conseqüência a desqualificação do ovo e em preços inferiores para os produtores.

A vitamina D é essencial para manter a produção de ovos, a formação da casca e a homeostase do cálcio. O colecalciferol (vitamina $\mathrm{D}_{3}$ ) é produzido pela irradiação do 7-dehidrocolesterol, o qual é sintetizado no corpo e se desloca até a camada externa da pele. O colecalciferol, após a absorção, é transportado ao fígado onde é hidroxilado na posição 25, originando o metabólito 25-hidroxicolecalciferol $\left(25(\mathrm{OH}) \mathrm{D}_{3}\right)$, o qual é direcionado aos rins e hidroxilado no carbono 1 originando o metabólito ativo 1,25-dihidroxicolecalciferol $\left(1,25(\mathrm{OH})_{2} \mathrm{D}_{3}\right)$, que exerce funções metabólicas chamadas nuclear e não-nuclear (Leeson \& Summers, 2001; Pesti et al., 2005). Poedeiras que produzem ovos com cascas normais possuem maior atividade da enzima 1- hidroxilase renal e concentrações plasmáticas de $1,25(\mathrm{OH})_{2} \mathrm{D}_{3}$ e de calbindina duodenal e uterina superiores às de poedeiras que produzem ovos sem casca. No entanto, as concentrações plasmáticas de cálcio são similares (Bar et al., 1999). Foi constatado por Yoshimura et al. (1997) que os receptores de vitamina D estão mais concentrados no útero que em outros segmentos do oviduto de poedeiras em produção. De Luca (1972) e Soares et al. (1995) relataram que o metabólito $25(\mathrm{OH}) \mathrm{D}_{3}$ é de 2,5 a 4,5 vezes mais ativo que o colecalciferol, portanto, é de grande valia na prevenção de problemas ósseos e de espessura da casca dos ovos. Trabalhos mais recentes (Leeson \& Summers, 2001) indicam que o $25(\mathrm{OH}) \mathrm{D}_{3}$ pode ser 200 vezes mais efetivo que o colecalciferol na absorção intestinal de cálcio. Os níveis sugeridos de vitamina D para poedeiras variam de 300 a 2.500 UI/kg de ração (NRC, 1994; Leeson \& Summers, 1997, 2001; Feedstuffs, 1998; Rostagno et al., 2005).

De acordo com Leeson \& Summers (2001), a vitamina C é requerida somente para humanos, primatas, cobaias e peixes, pois na maioria das outras espécies é sintetizada em quantidades adequadas. Tem-se relatado que a suplementação de dietas com vitamina C melhora a condição dos ossos da perna em aves estressadas (Leeson \& Summers, 2001). Nessas condições, não se sabe exatamente se a vitamina $C$ é benéfica na síntese de pré-colágeno, um precursor da formação óssea, ou se atua mais diretamente na ativação da enzima 1 - hidroxilase renal, assim, a conversão de $25(\mathrm{OH}) \mathrm{D}_{3}$ para $1,25(\mathrm{OH})_{2} \mathrm{D}_{3} \mathrm{e} 24,25(\mathrm{OH})_{2} \mathrm{D}_{3}$ depende da suplementação de vitamina C. Essa hipótese foi explorada por Keshavarz (1996), o qual relatou que a vitamina C pode estar envolvida na hidroxilação do metabólito $25(\mathrm{OH}) \mathrm{D}_{3}$ para $1,25(\mathrm{OH})_{2} \mathrm{D}_{3}$. Assim, é provável alguma resposta benéfica da vitamina $\mathrm{C}$ quando se fizer uso do metabólito $25(\mathrm{OH}) \mathrm{D}_{3}$ como substrato.
Considerando a importância metabólica das vitaminas D e C, objetivou-se avaliar os efeitos da suplementação de duas fontes de vitamina D (colecalciferol e 25hidroxicolecalciferol) em associação à vitamina $\mathrm{C}$ sobre o desempenho, a qualidade interna e externa dos ovos, as concentrações plasmáticas de cálcio total e iônico e as características ósseas de poedeiras comerciais na fase inicial de produção.

\section{Material e Métodos}

Foram utilizadas 288 galinhas da linhagem comercial ISA Babcock B300 ${ }^{\circledR}$ com 23 semanas de idade, alojadas em pares em gaiolas metálicas medindo $0,25 \mathrm{~m} \times 0,40 \mathrm{~m} \times 0,40 \mathrm{~m}$ (frente, profundidade e altura, respectivamente) por um período de 12 semanas. As aves foram submetidas a fotoperíodos crescentes (de 20 a 28 semanas) e constantes (16 horas de luz/dia, a partir das 29 semanas).

O delineamento experimental foi o inteiramente ao acaso em arranjo fatorial $2 \times 3$, com os fatores: fontes de vitamina $\mathrm{D}$ (colecalciferol e $25(\mathrm{OH}) \mathrm{D}_{3}$ ) e níveis de suplementação de vitamina C (0,100 e 200 ppm), totalizando seis combinações com oito repetições de seis aves. A ração basal (Tabela 1) foi formulada visando atender às exigências nutricionais para a idade/fase de produção das aves, conforme a composição química e os valores energéticos dos ingredientes descritos por Rostagno et al. (2000). À ração basal foi adicionada uma pré-mistura (Rovimix $\mathrm{D}^{\circledR}$ ) de vitamina $\mathrm{D}$, na forma de colecalciferol, proporcionando o nível de 2.756 UI de vitamina $\mathrm{D}_{3} / \mathrm{kg}$ de ração, e o equivalente na forma do metabólito 25-hidroxicolecalciferol $\left(25(\mathrm{OH}) \mathrm{D}_{3}\right)$ com a inclusão de $5,51 \mathrm{~g}$ do produto comercial Hy. $\mathrm{D}^{\circledR}$ por tonelada de ração, combinados com 0, 100 ou 200 ppm de vitamina C (Rovimix Stay C $35^{\circledR}$ ). Adotou-se um período pré-experimental de 10 dias, no qual as aves foram alimentadas com as rações experimentais. Ração e água de bebida foram fornecidas à vontade por todo o período experimental. Foram avaliadas as características de desempenho, como consumo de ração (g/ave/dia), conversão alimentar (g ração/ g ovo), produção de ovos (\% ovos/ave/dia), peso dos ovos (g) e massa de ovos (g/ave/dia). Semanalmente, a produção total de um dia foi pesada para obtenção do peso médio dos ovos. A massa de ovos foi obtida pela multiplicação do percentual de produção de ovos pelo peso médio dos ovos. As características de qualidade interna dos ovos avaliadas foram as porcentagens de albúmen e gema, obtidas com a utilização de todos os ovos produzidos nos últimos dois dias de cada período experimental.

Também foram avaliadas as características de qualidade externa dos ovos, como porcentagem de casca, espessura de 
Tabela 1 - Composição percentual e níveis nutricionais calculados da ração basal

\begin{tabular}{lc}
\hline Ingrediente & $(\%)$ \\
\hline Milho moído & 54,51 \\
Farelo de soja 45\% & 30,80 \\
Calcário calcítico & 8,36 \\
Fosfato bicálcico & 1,88 \\
Óleo de soja & 3,80 \\
Sal & 0,39 \\
DL-metionina 99\% & 0,01 \\
Suplemento vitamínico e mineral* & 0,25 \\
Total & 100,00 \\
\hline Energia e nutrientes calculados (\%) & \\
\hline Energia metabolizável (kcal/kg) & 2870 \\
Proteína bruta & 18,71 \\
Cálcio & 3,80 \\
Fósforo disponível & 0,45 \\
Sódio & 0,19 \\
Lisina total & 0,99 \\
Metionina total & 0,30 \\
Metionina + cistina total & 0,60 \\
Ácido linoléico & 3,31 \\
\hline
\end{tabular}

* Conteúdo por kg de dieta: vit. A - 6250 UI; vit. E - 15 UI; vit. K - 0,04 mg; vit. $\mathrm{B}_{1}$ - 0,25 mg; vit. $\mathrm{B}_{2}-3,4 \mathrm{mg}$; vit. $\mathrm{B}_{6}-0,25 \mathrm{mg}$; vit. $\mathrm{B}_{12}-20 \mathrm{mcg}$; ácido pantotênico - 3,8 mg; niacina - 9,9 mg; biotina - 0,1 mg; ácido fólico - 0,25 mg; Cu - 6 mg; Fe - 52,5 mg; I - 0,33 mg; Se - 0,21 mg; Mn - 48 mg; Zn - 60,23 mg; metionina - $1.250 \mathrm{mg}$; antioxidante - 0,313 mg; veículo q.s.p. - $1.000 \mathrm{~g}$.

casca (mm) e peso específico do ovo $\left(\mathrm{g} / \mathrm{mL} \mathrm{H}_{2} \mathrm{O}\right)$, com a utilização de todos os ovos produzidos nos últimos dois dias de cada período experimental e as concentrações de cálcio sérico total e iônico (mg/dL), com a utilização de kit comercial.

Após o sacrifício de duas aves por unidade experimental, ambas as tíbias foram retiradas. A esquerda foi congelada (contraprova) e a direita, dissecada para posteriores análises. As tíbias foram secas em estufa de ventilação forçada a $22^{\circ} \mathrm{C}$, em ambiente com temperatura e umidade controladas, por sete dias. Características físicas ósseas foram determinadas pelo teste de três pontos comumente utilizados para avaliar a resistência óssea (Crenshaw et al., 1981). As medidas externas do osso (diâmetro) foram determinadas em dois pontos medianos usando um paquímetro eletrônico, uma no plano perpendicular à direção em que a força foi aplicada e uma paralela à força. O teste de resistência foi conduzido usando um Texturômetro Texture Analyser TAXT2 com um movimento de haste de $5 \mathrm{~mm} /$ minuto e com carga de $25 \mathrm{~kg}$. Os ossos foram apoiados com a parte dorsal para cima sobre um suporte de $6,5 \mathrm{~cm}$. A força máxima para a quebra e a deformação foram determinadas para cada tíbia direita. Depois que os ossos foram quebrados, as espessuras das paredes foram determinadas, novamente perpendicular e paralela à força aplicada. A tensão (megapascals) e módulo de elasticidade (gigapascals) foram gerados pelo sistema computadorizado do texturômetro utilizado. Os fragmentos dos ossos foram então recolhidos, macerados e submetidos à extração de gordura em éter de petróleo por 8 horas, para análise de cinzas (\%) e cálcio (\% de cinzas) ósseo. Foram então secos em estufa por 12 horas e levados a mufla por 12 horas a $600^{\circ} \mathrm{C}$ para determinação da quantidade de cálcio por espectrofotometria de absorção atômica (AOAC, 1980).

Durante o período experimental foram registradas as temperaturas máxima e mínima em três horários do dia (8 h, $12 \mathrm{~h}$ e $16 \mathrm{~h}$ ). As temperaturas médias foram $24,45^{\circ} \mathrm{C} \pm 1,85$ e $18,22^{\circ} \mathrm{C} \pm 3,96$ para a máxima e mínima, respectivamente. A umidade relativa do ar média foi de 63,97\% \pm 13,58.

Os dados obtidos foram submetidos à análise estatística pelo Programa de Análises Estatísticas (Estat 2.0, 1992) e as médias comparadas pelo teste Tukey no nível de 5\% de probabilidade.

\section{Resultados e Discussão}

Os dados de consumo de ração, produção, peso e massa de ovos não foram influenciados $(\mathrm{P}>0,05)$ pelos fatores estudados (Tabela 2). No entanto, para a conversão alimentar, verificou-se interação $(P<0,01)$ entre os fatores (Tabela 3). Os melhores valores para a conversão alimentar foram obtidos quando as galinhas foram alimentadas com rações suplementadas com vitamina C (100 ou 200 ppm) em associação ao colecalciferol ou com a utilização do metabólito $25(\mathrm{OH}) \mathrm{D}_{3}$ sem a inclusão de vitamina C.

Houve interação entre os fatores para as porcentagens de albúmen $(\mathrm{P}<0,01)$ e de gema $(\mathrm{P}<0,05)$ (Tabela 3$)$. A suplementação das dietas com 200 ppm de vitamina C e colecalciferol proporcionou maior percentual de albúmen em comparação às dietas não suplementadas com vitamina C ou suplementadas com o metabólito $25(\mathrm{OH}) \mathrm{D}_{3}$ (Tabela 3 ). Por outro lado, a utilização do metabólito $25(\mathrm{OH}) \mathrm{D}_{3}$ em associação a 200 ppm de vitamina C proporcionou maior percentual de gema.

Para a porcentagem e a espessura de casca também ocorreram interações $(\mathrm{P}<0,01)$ entre os fatores (Tabela 3$)$. $\mathrm{O}$ maior percentual de casca foi observado quando as dietas foram suplementadas com 200 ppm de vitamina C em associação ao metabólito $25(\mathrm{OH}) \mathrm{D}_{3}$. A suplementação de 100 ppm de vitamina C em associação ao metabólito $25(\mathrm{OH}) \mathrm{D}_{3}$ proporcionou maior espessura de casca em comparação ao uso do colecalciferol. Na ausência de vitamina $\mathrm{C}$, os valores de porcentagem e espessura de casca foram semelhantes, independentemente da fonte de vitamina D utilizada.

Esses resultados coincidem com os relatos de Hamilton (1980), que também não encontraram diferenças no consumo de ração, na produção e no peso de ovos ao trabalharem 
Tabela 2 - Desempenho de poedeiras alimentadas com rações com diferentes fontes de vitamina D e níveis de vitamina C

\begin{tabular}{|c|c|c|c|c|c|c|}
\hline \multirow[t]{2}{*}{ Característica } & \multicolumn{2}{|c|}{ Fonte vitamina D } & \multicolumn{3}{|c|}{ Nível vitamina C (ppm) } & \multirow[t]{2}{*}{ CV (\%) } \\
\hline & Colecalciferol & $25(\mathrm{OH}) \mathrm{D}_{3}$ & 0 & 100 & 200 & \\
\hline Consumo de ração (g) & 90,12 & 90,12 & 90,80 & 90,00 & 89,55 & 4,27 \\
\hline Produção de ovos (\%) & 91,65 & 92,45 & 90,84 & 92,37 & 92,94 & 4,32 \\
\hline Peso ovos (g) & 58,97 & 58,17 & 58,60 & 58,37 & 58,75 & 2,61 \\
\hline Massa ovos (g/ave/dia) & 54,14 & 53,85 & 53,31 & 54,02 & 54,65 & 4,79 \\
\hline Conversão alimentar* & 1,67 & 1,68 & 1,71 & 1,67 & 1,64 & 4,66 \\
\hline
\end{tabular}

* Houve interação significativa $(\mathrm{p}<0,05)$ e o desdobramento das combinações fontes de vitamina D e níveis de vitamina C encontra-se na Tabela 3 .

Tabela 3 - Conversão alimentar e características de qualidade interna e externa de ovos de poedeiras alimentadas com rações com diferentes fontes de vitamina $\mathrm{D}$ e níveis de vitamina $\mathrm{C}$

\begin{tabular}{|c|c|c|c|}
\hline \multirow[t]{2}{*}{ Fonte vitamina $\mathrm{D}$} & \multicolumn{3}{|c|}{ Nível vitamina C (ppm) } \\
\hline & 0 & 100 & 200 \\
\hline & \multicolumn{3}{|c|}{ Conversão alimentar } \\
\hline Colecalciferol & $1,76 \mathrm{Aa}$ & $1,65 \mathrm{Ba}$ & $1,61 \mathrm{Ba}$ \\
\hline \multirow[t]{2}{*}{$25(\mathrm{OH}) \mathrm{D}_{3}$} & $1,66 \mathrm{Ab}$ & $1,70 \mathrm{Aa}$ & $1,68 \mathrm{Aa}$ \\
\hline & \multicolumn{3}{|c|}{ Porcentagem de albúmen } \\
\hline Colecalciferol & $59,86 \mathrm{Ba}$ & $60,52 \mathrm{ABa}$ & $61,13 \mathrm{Aa}$ \\
\hline \multirow[t]{2}{*}{$25(\mathrm{OH}) \mathrm{D}_{3}$} & $60,65 \mathrm{Aa}$ & $60,26 \mathrm{Aa}$ & $59,78 \mathrm{Ab}$ \\
\hline & \multicolumn{3}{|c|}{ Porcentagem de gema } \\
\hline Colecalciferol & $24,83 \mathrm{Aa}$ & $24,68 \mathrm{Aa}$ & $24,37 \mathrm{Ab}$ \\
\hline \multirow[t]{2}{*}{$25(\mathrm{OH}) \mathrm{D}_{3}$} & $24,44 \mathrm{Ba}$ & $24,68 \mathrm{ABa}$ & $25,27 \mathrm{Aa}$ \\
\hline & \multicolumn{3}{|c|}{ Porcentagem de casca } \\
\hline Colecalciferol & $9,81 \mathrm{Aa}$ & $9,68 \mathrm{ABa}$ & $9,44 \mathrm{Bb}$ \\
\hline \multirow[t]{2}{*}{$25(\mathrm{OH}) \mathrm{D}_{3}$} & 9,61Аa & $9,64 \mathrm{Aa}$ & 9,70Aa \\
\hline & \multicolumn{3}{|c|}{ Espessura de casca $(\mathrm{mm})$} \\
\hline Colecalciferol & $0,44 \mathrm{Aa}$ & $0,42 \mathrm{Bb}$ & $0,42 \mathrm{Ba}$ \\
\hline $25(\mathrm{OH}) \mathrm{D}_{3}$ & $0,43 \mathrm{Aa}$ & $0,44 \mathrm{Aa}$ & $0,43 \mathrm{Aa}$ \\
\hline
\end{tabular}

Médias seguidas de mesma letra maiúscula (linha) e minúscula (coluna) não diferem $(\mathrm{p}>0,05)$ entre si pelo teste Tukey.

com colecalciferol e $25(\mathrm{OH}) \mathrm{D}_{3}$ em ração para poedeiras. Outro achado diferente foi obtido por Terry et al. (1999), que verificaram melhor peso do ovo quando as galinhas foram alimentadas com o metabólito $25(\mathrm{OH}) \mathrm{D}_{3}$. O peso específico dos ovos (valor médio de $1,090 \mathrm{~g} / \mathrm{mL} \mathrm{H}_{2} \mathrm{O}$ ) não foi influenciado pelos fatores estudados em todo período experimental. Estes achados coincidem com os resultados obtidos por Roland \& Harms (1976), que utilizaram o metabólito $25(\mathrm{OH}) \mathrm{D}_{3}$, sem adição de vitamina $\mathrm{C}$, e não encontraram melhora na qualidade de casca de poedeiras jovens.

Keshavarz (1996), entretanto, ao avaliar os efeitos da utilização de altos níveis de vitamina $\mathrm{C}$ na presença de $25(\mathrm{OH}) \mathrm{D}_{3}$ e colecalciferol sobre as cinzas ósseas e a qualidade de casca de ovos de poedeiras, não verificou efeito interativo entre as variáveis estudadas. Neste estudo, a porcentagem e a espessura de casca diminuíram à medida que se aumentou o nível de vitamina C, na presença de colecalciferol. Em condições criatórias bastante satisfatórias, a utilização de níveis de colecalciferol acima de 2.500 UI/kg de ração com a suplementação de vitamina C (200 ou 400 ppm) mostrou-se sem efeito expressivo sobre o desempenho, a qualidade interna e externa dos ovos e os níveis séricos de cálcio total e iônico (Faria et al., 1999a). Por outro lado, quando as aves foram criadas na estação de verão (Faria et al., 1999b), na primavera (Faria et al., 1999c) ou em condições de estresse calórico (Faria et al., 2001), a utilização de níveis mais elevados de colecalciferol (3.000 ou 3.500 UI/kg de ração) e a suplementação de vitamina C (200 ou 400 ppm) proporcionaram melhoria nas características de desempenho e de qualidade da casca dos ovos. Poedeiras com 72 semanas de idade não manifestaram alteração no desempenho e na qualidade da casca dos ovos, quando foram alimentadas com 500 ou 2.500 UI de colecalciferol/kg de ração (Faria et al., 2000).

As características resistência da tíbia à quebra e a porcentagem de cinzas não foram afetadas pelas fontes de vitamina D nem pelos níveis de vitamina $\mathrm{C}$ avaliados (Tabela 4). A porcentagem de cálcio nas cinzas foi influenciada pela fonte de vitamina $\mathrm{D}$, de modo que os maiores valores foram observados com a utilização de colecalciferol. Os valores médios dos diâmetros, interno $(0,50 \mathrm{~cm})$ e externo $(0,63 \mathrm{~cm})$ das tíbias não foram influenciados pelas fontes de vitamina D e pelos níveis de vitamina C.

Embora utilizando níveis de vitamina $\mathrm{C}$ bem superiores, Orban et al. (1993) trabalharam com suplementação de vitamina $\mathrm{C}$ em quantidades que variaram de 1.000 a 3.000 ppm e também não observaram melhora na resistência óssea de tíbias, apesar do significativo aumento linear no conteúdo mineral ósseo e na densidade óssea.

Avaliando o colecalciferol e o metabólito $1 \mathrm{aOHD}_{3}$, considerado mais ativo que o $25(\mathrm{OH}) \mathrm{D}_{3}$, Soares et al. (1988) observaram maior resistência de tíbia para as aves alimentadas com o metabólito $1 \mathrm{aOHD}_{3}$. Ressalta-se que esse metabólito não se encontra disponível comercialmente para alimentação animal. 
Em experimento realizado por Newman \& Leeson (1999), a suplementação de $100 \mathrm{ppm}$ de vitamina C para galinhas de 72 semanas não influenciou as características ósseas, como cálcio e cinzas ósseas, e a resistência de tíbia. Keshavarz (1996), avaliando colecalciferol e $25(\mathrm{OH}) \mathrm{D}_{3}$ na dosagem de 500 UI/kg, e ácido ascórbico variando de zero a 1.000 ppm, não encontrou alterações para cinzas ósseas.

Avaliando o colecalciferol e os metabólitos $25(\mathrm{OH}) \mathrm{D}_{3}$ e $1 \alpha \mathrm{OHD}_{3}$, Soares et al. (1983) não observaram diferenças nos teores de cálcio e cinzas ósseas. Em outro experimento, Soares et al. (1988) trabalharam somente com o colecalciferol e o metabólito $1 \alpha \mathrm{OHD}_{3}$ e constataram maior porcentagem de cálcio na tíbia e maior resistência óssea quando utilizaram o metabólito $1 \alpha \mathrm{OHD}_{3}$, o que evidencia a necessidade de constantes avaliações dos metabólitos da vitamina D em diferentes condições experimentais.

A substituição do colecalciferol $(1.000,2.000$ e $4.000 \mathrm{UI} / \mathrm{kg}$ de ração) pelo metabólito $25(\mathrm{OH}) \mathrm{D}_{3}$ em dietas para frangos de corte foi avaliada por Fritts \& Waldroup (2005), que constataram que o nível de $4.000 \mathrm{UI} / \mathrm{kg}$ melhorou o peso corporal de frangos aos 42 dias de idade. No entanto, as fontes de vitamina D e os níveis de vitamina C não influenciaram a conversão alimentar, a mortalidade, o teor de cinzas ósseas e o número de ossos quebrados durante o processamento automático das aves, o que tem relação com os achados neste estudo com poedeiras jovens (cinza óssea e resistência óssea à quebra).

As concentrações plasmáticas de cálcio total e iônico não sofreram influência dos níveis de vitamina avaliados (Tabela5).

Orban et al. (1993) encontraram aumento nos níveis de cálcio plasmático quando alimentaram frangos e galinhas com ácido ascórbico com rações contendo ácido ascórbico, o que, segundo os autores, é um indicativo do envolvimento da vitamina C na mobilização de cálcio pela acentuada absorção intestinal. Contudo, isto não foi observado neste estudo.

Tabela 4 - Resistência a quebra (kgf), cinzas (\%) e cálcio (\% das cinzas) de tíbia de poedeiras alimentadas com rações com diferentes fontes de vitamina D e níveis de vitamina C

\begin{tabular}{|c|c|c|c|c|c|c|}
\hline \multirow[t]{2}{*}{ Característica } & \multicolumn{2}{|c|}{ Fonte vitamina D } & \multicolumn{3}{|c|}{ Nível vitamina C (ppm) } & \multirow[t]{2}{*}{ CV (\%) } \\
\hline & Colecalciferol & $25(\mathrm{OH}) \mathrm{D}_{3}$ & 0 & 100 & 200 & \\
\hline Resistência à quebra & 23,25 & 21,69 & 23,26 & 22,31 & 21,84 & - \\
\hline Cinzas & 53,66 & 53,47 & 53,28 & 53,33 & 54,08 & 3,83 \\
\hline Cálcio & $35,55 a$ & $34,53 b$ & 35,16 & 35,14 & 34,83 & 4,44 \\
\hline
\end{tabular}

Médias seguidas pela mesma letra, em cada linha e em cada fator, não diferem $(\mathrm{P}>0,05)$ entre si pelo teste Tukey.

Tabela 5 - Níveis plasmáticos de cálcio total e iônico $(\mathrm{mg} / \mathrm{dL})$ de poedeiras alimentadas com rações com diferentes fontes de vitamina D e níveis de vitamina $\mathrm{C}$

\begin{tabular}{|c|c|c|c|c|c|c|}
\hline \multirow[t]{2}{*}{ Característica } & \multicolumn{2}{|c|}{ Fonte vitamina D } & \multicolumn{3}{|c|}{ Nível vitamina C (ppm) } & \multirow[t]{2}{*}{ CV (\%) } \\
\hline & Colecalciferol & $25(\mathrm{OH}) \mathrm{D}_{3}$ & 0 & 100 & 200 & \\
\hline Cálcio total & 7,50 & 7,66 & 7,89 & 7,27 & 7,58 & 13,21 \\
\hline Cálcio iônico & 5,22 & 5,25 & 5,50 & 4,98 & 5,23 & 13,61 \\
\hline
\end{tabular}

\section{Conclusões}

A conversão alimentar de poedeiras na fase inicial de produção melhora com a utilização de vitamina $\mathrm{D}$ sob a forma de $25(\mathrm{OH}) \mathrm{D}_{3}$. A suplementação de vitamina C (100 ou 200 ppm) em associação ao colecalciferol também proporciona melhor conversão alimentar em poedeiras jovens. A associação da vitamina $\mathrm{D}$ sob a forma de $25(\mathrm{OH}) \mathrm{D}_{3}$ com 200 ppm de vitamina C aumenta o percentual de gema dos ovos. A espessura e porcentagem de casca melhoram com a utilização de $25(\mathrm{OH}) \mathrm{D}_{3}$ e a suplementação de vitamina C nas dietas (100 ou 200 ppm, respectivamente).

\section{Literatura Citada}

ASSOCIATION OF OFFICIAL ANALYTICAL CHEMISTS - AOAC. Official methods of analysis. 3.ed. Washington D.C.: 1980.

BAR, A.; VAX, E.; STRIEM, S. Relationships among age, eggshell thickness and vitamin D metabolism and its expression in the laying hen. Comparative Biochemistry and Physiology, v.123, p.147-154, 1999 (Part A).

CRENSHAW, T.D.; PEO, E.R.; LEWIS, A.J. et al. Bone strength as a trait for assessing mineralization in swine: a critical review 
of techniques involved. Journal of Animal Science, v.53, p.827-835, 1981.

DE LUCA, H.F. Metabolites of vitamin D: new tools of medicine and nutrition. Proceedings of Cornell Nutrition Conference, p.20-24, 1972.

ESTAT 2.0 Sistema de análise estatística. Jaboticabal: Polo Computacional - Departamento de Ciências Exatas - UNESP; 1992. (CD-ROM).

FARIA, D.E.; JUNQUEIRA, O.M.; SAKOMURA, N.K. et al. Influência de diferentes níveis de energia, vitaminas $\mathrm{D}_{3}$ e relação sódio:cloro $\mathrm{C}$ sobre o desempenho e qualidade dos ovos de poedeiras comerciais. Revista Brasileira de Zootecnia, v.29, n.2, p.467-475, 2000

FARIA, D.E.; JUNQUEIRA, O.M.; SOUZA, P.A. et al. Suplementação de vitaminas D e C para poedeiras comerciais durante o primeiro ciclo de produção. Revista Brasileira de Ciência Avícola, v.1, n.2, p.135-144, 1999a.

FARIA, D.E.; JUNQUEIRA, O.M.; SOUZA, P.A. et al. Influência de diferentes níveis de vitaminas $\mathrm{D}$ e $\mathrm{C}$ e idade das galinhas poedeiras sobre o desempenho e qualidade dos ovos. 1 - Verão. Revista Brasileira de Ciência Avícola, v.1, n.3, p.193-201, 1999b.

FARIA, D.E.; JUNQUEIRA, O.M.; SOUZA, P.A. et al. Influência de diferentes níveis de vitaminas $\mathrm{D}$ e $\mathrm{C}$ e idade das galinhas poedeiras sobre o desempenho e qualidade dos ovos. 2 - Primavera. Revista Brasileira de Ciência Avícola, v.1, n.3, p.203-210, 1999c.

FARIA, D.E.; JUNQUEIRA, O.M.; SOUZA, P.A. et al. Desempenho, temperatura corporal e qualidade dos ovos de poedeiras alimentadas com vitaminas D e C em três temperaturas ambiente. Revista Brasileira de Ciência Avícola, v.3, n.1, p.49-56, 2001.

FEEDSTUFFS. Reference Issue, p.70, 1998.

FRITTS, C.A.; WALDROUP, P.W. Comparison of cholecalciferol and 25-hydroxycholecalciferol in broiler diets designed to minimize phosphorus excretion. Journal of Applied Poultry Research, v.14, p.156-166, 2005.

HAMILTON, R.M.G. The effects of dietary phosphorus, vitamin $\mathrm{D}_{3}$ and 25-hydroxyvitamin $\mathrm{D}_{3}$ levels on feed intake, productive performance, and egg and shell quality in two strains of force-molted white Leghorns. Poultry Science, v.59, p.598-604, 1980

KESHAVARZ, K. The effect of different levels of vitamin C and cholecalciferol with adequate or marginal levels of dietary calcium on performance and eggshell quality of laying hens. Poultry Science, v.48, p.1227-1235, 1996.
LEESON, S.; SUMMERS, J.D. Scott's nutrition of the chicken. 4.ed. Guelph: University Books, 2001. 591p.

LEESON, S.; SUMMERS, J.D. Commercial poultry nutrition. 2.ed. Guelph: University Books, 1997. 350p.

NATIONAL RESEARCH COUNCIL - NRC. Nutrient requirements of poultry. 9.rev.ed. Washington: National Academy Press, 1994. 155p.

NEWMAN, S.; LEESON, S. The effect of dietary supplementation with 1,25-dihydroxycholecalciferol or vitamin $C$ on the characteristics of the tibia of older laying hens. Poultry Science, v.78, p.85-90, 1999.

ORBAN, J.I.; ROLAND, SR.D.A.; CUMMINS, K. et al. Influence of large doses of ascorbic acid on performance, plasma calcium, bone characteristics, and eggshell quality in broilers and leghorn hens. Poultry Science, v.72, p.691-700, 1993.

PESTI, G.M.; BAKAlli, R.I.; DRIVER, J.P. et al. Poultry nutrition and feeding: a textbook. Athens: Trafford Publishing, 2005. paginação descontínua.

ROLAND, D.A.; HARMS, R.H. The lack of response of 25-hydroxyvitamin D on egg shell quality or other criteria in laying hens. Poultry Science, v.55, p.1083-1085, 1976.

ROSTAGNO, H.S.; ALBINO, L.F.T.; DONZELE, J.L. et al. Tabelas brasileiras para aves e suínos: composição de alimentos e exigências nutricionais. 2.ed. Viçosa, MG: Editora UFV, 2000. $141 \mathrm{p}$.

ROSTAGNO, H.S.; ALBINO, L.F.T.; DONZELE, J.L. et al. Tabelas brasileiras para aves e suínos: composição de alimentos e exigências nutricionais. 3.ed. Viçosa, MG: Editora UFV, 2005. $186 \mathrm{p}$.

SOARES, JR., J.H.; KAETZEL, D.M.; ALLEN, J.T. et al. Toxicity of a vitamin D steroid to laying hens. Poultry Science, v.62, p.24-29, 1983.

SOARES, JR., J.H.; OTTINGER, M.A.; BUSS, E.G. Potential role 1,25-dihydroxycholecalciferol in egg shell calcification. Poultry Science, v.67, p.1322-1328, 1988.

SOARES JR., J.H.; KERR, J.M.; GRAY, R.W. 25-hydroxycholecalciferol in poultry nutrition. Poultry Science, v.74, p.1919-1934, 1995.

TERRY, M.; LANENGA, M.; McNAUGHTON, J.L. et al. Safety of 25hydroxyvitamin $\mathrm{D}_{3}$ as a source of vitamin $\mathrm{D}_{3}$ in layer poultry feed. Veterinary and Human Toxicology, v.41, p.312-316, 1999.

YOSHIMURA, Y.; OHIRA, H.; TAMURA, T. Immunocytochemical localization of vitamin D receptors in the shell gland of immature, laying, and molting hens. General and Comparative Endocrinology, v.108, p.282-289, 1997. 